\title{
The challenge of cardiovascular prevention in NAFLD
}

Non-alcoholic fatty liver disease (NAFLD) has traditionally been interpreted as a liver disease with high risk of developing liver-related complications and most patients have usually been referred to gastroenterologists and hepatologists. However, patients also have an increased chance of developing cardiovascular diseases and the landscape of cardiovascular risk in this clinical setting is continuously evolving.

New evidence on this topic has been recently added by a comprehensive review and meta-analysis in The Lancet Gastroenterology \& Hepatology. ${ }^{1}$ This analysis included more than 5 million middle-aged individuals with 99668 incident cases of fatal and non-fatal cardiovascular events, confirming that NAFLD is associated with a moderately increased risk of fatal or non-fatal cardiovascular events (pooled hazard ratio [HR] 1.45, 95\% Cl 1.31-1.61), with this risk increasing in patients with established fibrosis $(2.50,1.68-3.72)$. The authors also performed meta-regression analyses and found that this association is not modified by age, bodymass index (BMI), and sex, while it seems to be more evident in studies with a high proportion of participants with diabetes and to be linear with LDL-cholesterol. Despite the large sample of patients included, the results should be interpreted with caution given the high heterogeneity of the included studies and the presence of some unexplored confounding factors.

NAFLD includes different clinical phenotypes and not all patients will develop cardiovascular disease. How can we identify those at higher risk? This is a crucial open issue in the clinical management of patients with NAFLD. Mantovani and colleagues ${ }^{1}$ reported higher incidence of fatal and non-fatal cardiovascular events in patients with more severe liver fibrosis. Interestingly, this finding was obtained by pooling data from studies diagnosing fibrosis with both liver biopsy and noninvasive markers. Recently, a significant association between non-invasive markers of liver fibrosis (FIB-4 and NAFLD fibrosis score) and cardiovascular outcomes in patients with NAFLD was observed in the prospective Plinio study. ${ }^{2}$ Advanced liver fibrosis is common in patients with type 2 diabetes, who need to be screened routinely with non-invasive markers of fibrosis. Finally, it has also been proposed that hepatic fibrosis should be considered as a new player in the stratification of residual cardiovascular risk. ${ }^{3}$

An interesting finding of the meta-analysis is that the association between NAFLD and cardiovascular events was not modified by BMI. This finding might be explained by the fact that most of the studies included participants who were overweight or obese. As such, patients with lean NAFLD, who usually have genetically driven NAFLD, seem to have more liver-related than cardiovascular-related complications. ${ }^{4}$ Cardiovascular risk in these patients needs further attention.

Cardiovascular prevention should start with a programme of extensive lifestyle intervention. The Mediterranean diet is the best cardioprotective dietary approach, with benefits on insulin resistance ${ }^{5}$ and oxidative stress ${ }^{6}$ having been reported in patients with NAFLD. Particular attention must be paid to the treatment of overweight and obesity, which are the most important predisposing factors for the development of NAFLD. Weight loss is highly recommended in most patients and improves liver histology, insulin resistance, and low-grade chronic inflammation?

A further step is to treat the so-called atherogenic dyslipidaemia present in most patients with NAFLD. The main objective of such therapy is the achievement of risk-related LDL-cholesterol targets. Results of this meta-analysis seem to further support the role of LDL in NAFLD. Statins constitute the main drug therapy, although they are often under prescribed due to concerns regarding possible liver toxicity. ${ }^{8}$ However, statins were proved to be safe in patients with NAFLD. ${ }^{9}$ Moreover, possible beneficial effects of statins on liver histology have been suggested in patients with diabetes and NAFLD..$^{10}$

Finally, given that cardiovascular disease is the main cause of morbidity and mortality in NAFLD, cardiovascular costs should also be taken into consideration, which are no less that those related to management of liver disease, greatly increasing the already high economic burden of NAFLD.

These findings strongly suggest the need for a better definition of the objectives of clinical approaches to patients with NAFLD, who need more holistic approaches than those usually undertaken by
Lancet Gastroenterol Hepato 2021

Published Online September 22, 2021 https://doi.org/10.1016/ S2468-1253(21)00337-X See Online/Articles https://doi.org/10.1016/ PII 
medical specialists [A: rewording ok?] $]_{\mathbf{z}}$ In addition to hepatoprotection, cardiovascular prevention must be the main intervention strategy for patients with NAFLD. Cardiovascular risk assessment and treatment of comorbidities and risk factors must become a priority in the care of these patients. Gastroenterologists, hepatologists, diabetologist, internists, cardiologists, and nutritionists should act together to provide comprehensive care for patients with NAFLD.

In conclusion, Mantovani and colleagues ${ }^{1}$ again draw attention to the high cardiovascular risk in patients with NAFLD and place further emphasis on a major challenge for NAFLD management [A: rewording ok? $]_{2}$ the need of a more holistic approach, rather than a liver-centred approach.

We declare no competing interests.

*Maria Del Ben, Francesco Baratta, Daniele Pastori, Francesco Angelico maria.delben@uniroma1.it

[A:pleasegive fullaffliation dails for allauthers]

1 Mantovani A, Csermely A, Petracca G, et al. Non-alcoholic fatty liver disease and increased risk of fatal and nonfatal cardiovascular events: an updated systematic review and meta-analysis Lancet Gastroenterol Hepatol 2021 [ED TO UPDATE].
2 Baratta F, Pastori D, Angelico F, et al. Nonalcoholic fatty liver disease and fibrosis associated with increased risk of cardiovascular events in a prospective study. Clin Gastroenterol Hepatol 2019 [A: please provide volume and page

3 Angelico F, Baratta F, Pastori D, Ben MD. Assessment of hepatic fibrosis in MAFLD: a new player in the evaluation of residual cardiovascular risk? Dig Liver Dis 2021; 53: 383-84.

4 Fracanzani AL, Petta S, Lombardi R, et al. Liver and cardiovascular damage in patients with lean nonalcoholic fatty liver disease, and association with visceral obesity. Clin Gastroenterol Hepatol 2017; 15: 1604-11.

5 Baratta F, Pastori D, Polimeni L, et al. Adherence to Mediterranean diet and non-alcoholic fatty liver disease: effect on insulin resistance. Am J Gastroenterol 2017; 112: 1832-39.

6 Baratta F, Pastori D, Bartimoccia S, et al. Poor adherence to Mediterranean diet and serum lipopolysaccharide are associated with oxidative stress in patients with non-alcoholic fatty liver disease. Nutrients 2020; 12: [A: page

7 EASL-EASD-EASO Clinical Practice Guidelines for the management of nonalcoholic fatty liver disease. J Hepatol 2016; 64: 1388-402.

8 Del Ben M, Baratta F, Polimeni L, et al. Under-prescription of statins in patients with non-alcoholic fatty liver disease. Nutr Metab Cardiovasc Dis 2017; 27: 161-67.

9 Pastori D, Pani A, Di Rocco A, et al. Statin liver safety in non-alcoholic fatty liver disease: a systematic review and metanalysis. BrJ Clin Pharmacol 2021 [A: please provide volume and page numbers. If not available, please give date published online and $\mathrm{DO}]_{\text {. }}$

10 Nascimbeni F, Aron-Wisnewsky J, Pais R, et al. Statins, antidiabetic medications and liver histology in patients with diabetes with nonalcoholic fatty liver disease. BMJ Open Gastroenterol 2016; 3: e000075. 